\title{
Familial influences on the clinical characteristics of major depression: a twin study
}

Kendler KS, Neale MC, Kessler RC, Heath AC, Eaves LJ. Familial influences on the clinical characteristics of major depression: a twin study. Acta Psychiatr Scand 1992: 86: 371-378. (c) Munksgaard 1992.

We sought in this study to clarify the role that familial factors play in influencing the clinical presentation of major depression (MD). We examined the similarity of the historical and symptomatic features of MD in 176 pairs of female-female monozygotic (MZ) and dizygotic (DZ) twins from a population-based registry, where both members reported a history of MD defined by DSM-III-R criteria. The age at onset and treatment-seeking were significantly correlated in all twin pairs and the correlation in concordant $\mathrm{DZ}$ pairs was actually somewhat higher than in concordant $\mathrm{MZ}$ twins. The degree of impairment was modestly correlated in all twin pairs with substantially higher correlations in $\mathrm{MZ}$ vs DZ twins. No twin resemblance was observed for number of episodes or longest duration of an episode. Twin resemblance for the clinical features of MD was modest, but so was their consistency for the same individual over successive 1-year periods. However, in 5 of the 6 neurovegetative symptoms involving changes in appetite, weight and sleep, $M Z$ twins were significantly correlated and correlations were significantly greater in concordant $\mathrm{MZ}$ vs $\mathrm{DZ}$ twins. Although the familial factors that cause twin resemblance for the age at onset and treatment seeking appear to be largely environmental, twin resemblance for the degree of impairment and neurovegetative symptoms are probably due largely to genetic factors. Our results suggest that familial factors influence the predisposition to some clinical features of MD.

\author{
K. S. Kendler ${ }^{1,2}$, M. C. Neale ${ }^{2}$, \\ R. C. Kessler ${ }^{3}$, A. C. Heath ${ }^{4}$, \\ L. J. Eaves ${ }^{1,2}$ \\ Departments of ${ }^{1}$ Psychiatry and ${ }^{2}$ Human \\ Genetics, Medical College of Virginia/Virginia \\ Commonwealth University, Richmond, Virginia, \\ ${ }^{3}$ Institute for Social Research, University of \\ Michigan, Ann Arbor, Michigan, ${ }^{4}$ Department \\ of Psychiatry, Washington University School of \\ Medicine, St. Louis, Missouri, USA
}

Key words: depression; twin; family Dr. K.S. Kendler, Department of Psychiatry, Box 710 MCV Station, Richmond, VA 23298-0710, USA

Accepted for publication July 11, 1992
Major depression (MD) has consistently been shown to aggregate (or run) in families (1). Studies have also examined whether certain clinical characteristics of MD predict the risk of illness in relatives, including age at onset $(2-4)$, recurrence $(5,6)$, severity of impairment (5), number or kind of depressive symptoms $(7,8)$ and comorbidity $(2,9)$. Much less frequently examined has been the degree to which specific historical and clinical characteristics of MD are themselves familial (10-13). That is, when pairs of relatives are both affected with MD, how similar are the clinical features of their disorders?

The answer to such a question is of interest because it may clarify the nature of the familial influences on MD. For example, the familial influences on MD may be nonspecific in that they influence overall risk for MD but play no role in shaping the features of MD once an individual becomes affected. This hypothesis predicts that the clinical features of pairs of relatives both affected with MD will be uncorrelated. By contrast, the familial factors for MD could be specific in that they influence both the over- all liability to illness and the clinical features of the disorder in affected individuals. If true, this would predict considerable resemblance in pairs of affected relatives for the specific clinical features of MD.

We examined this question in 176 pairs of femalefemale twins from a population-based twin register in which both members reported, at some point in their lives, one or more episodes of MD meeting DSM-III-R criteria (14). Although limited by small sample size, a comparison of the correlations in concordant MZ vs DZ twins can begin to provide some insight into whether an observed familial resemblance for the clinical features of MD is the result of familial-environmental or genetic factors.

\section{Material and methods}

The data for this report come from a study of genetic and environmental risk factors for common mental disorders in Caucasian female same-sex twin pairs from the Virginia Twin Registry (15). The study was restricted to women, who were chosen because they 


\section{Kendler et al.}

would, compared to men, have substantially higher prevalence rates for mood and anxiety disorders. The Virginia Twin Registry is a population-based register formed from a systematic review of all birth certificates, from 1918 onwards, in the Commonwealth of Virginia. The current addresses are obtained largely by matching to state records.

Twins were eligible to participate in this study if they were born between 1934 and 1971 and both members of the pair had previously responded to a mailed questionnaire, to which the individual response rate was approximately $64 \%$. The cooperation rate was almost certainly higher than this, as an unknown number of twins did not receive their questionnaire due to faulty addresses, improper forwarding of mail, etc. Of the total 1176 eligible pairs, neither twin was successfully interviewed in 46 , one twin was interviewed and the other refused in 97 and interviews were completed with both members of 1033 twin pairs. The individual cooperation rate was $92 \%$. Of the completed interviews, $89 \%$ were completed face to face, nearly all in the twin's home, and $11 \%$ (mostly twins living outside Virginia) were interviewed by telephone. The mean age $( \pm S D)$ of the sample at first interview was $30.1 \pm 7.6$ and ranged from 17 to 55 . Zygosity was determined blindly by standard questions (16), photographs, and when necessary, DNA (17).

Attempts were made to recontact all 2163 originally interviewed twins a minimum of 1 year after their first assessment. We completed a second interview in $2001(93 \%)$ of them. Unlike the first interview, nearly all of the second ones $(99 \%)$ were completed by phone. Both interviews were conducted by trained social workers, blind to the status of the co-twin.

The first interview contained an adapted version of the Structured Interview for DSM-III-R (18) to assess the lifetime history of MD up to 1 year prior to the interview. In addition, both interviews inquired of all respondents whether, in the last year, they had experienced 20 specific mental symptoms, including all of the DSM-III-R criteria for MD (14). In each of these assessments, we disaggregated the 9 individual symptomatic criteria for MD in DSM-III-R, assessing separately weight loss, decreased appetite, weight gain, increased appetite, insomnia, hypersomnia, psychomotor agitation and psychomotor retardation. Together with depressed mood, loss of interest or pleasure, fatigue or tiredness, feelings of worthlessness or guilt, trouble thinking or concentrating and suicidal ideation, this meant that we assessed 14 individual depressive symptoms. However, as nearly all depressed individuals endorsed depressed mood, this item was insufficiently variable to be usefully analyzed. In addition to symptoms, individuals who met 3 or more symptomatic criteria were asked an additional series of questions that included: age at first episode, total number of episodes, duration of longest episode, degree of impairment during worst episode (scored as severe impairment or incapacitation, moderate impairment and no impairment) and treatment-seeking. Treatmentseeking was assessed by the question "During any of these times, did you seek professional help for the way you were feeling?". Because of their expected rarity in a general population sample, psychotic symptoms during the depressive episode were not assessed.

Interrater reliability for the first interview was assessed in 53 jointly conducted interviews. For the DSM-III-R diagnosis of MD, reliability was very high $(\kappa=0.96 \pm 0.04)$. In the 18 cases where both raters agreed on the presence of $\mathrm{MD}$, the individual symptoms were rated as present or absent with high reliability $(\kappa=0.98 \pm 0.02)$.

DSM-III-R criteria for MD were assessed by computer algorithm. MD was not diagnosed when somatic illness or drugs were likely causes of the depressive syndrome or when the depressive syndrome represented normal grief.

Each twin was assessed for MD for 3 time periods: lifetime prior to 1 year before the first interview, 1 year prior to the first interview and 1 year prior to the second interview. Age at onset was defined as the youngest age at which she reported an episode at any assessment. Longest duration was defined as the longest duration reported at any assessment. Impairment was defined as the most severe impairment reported at any assessment. The number of episodes was the total reported episodes summed over all 3 assessments. We also analyzed this variable as a dichotomy, termed recurrence, separating twins with only 1 lifetime episode of illness versus those with more than one. Treatment-seeking was scored present if it was reported at any assessment. The number of DSM-III-R criteria was chosen from the interview with the highest number of endorsed criteria. For individual symptoms in individuals with more than one episode, we used 2 rules for choosing which episode to examine: the one with the most symptoms or the one with the greatest impairment. In each case, ties were broken by taking the more recent episode. The overall results were quite similar for the 2 methods and we here report results from the episode with the most symptoms.

In this article, we use the term clinical features to refer to the following variables: age at onset, duration of the longest episode, impairment during worst episode, total number of episodes, recurrence, treatment-seeking and number of DSM-III-R criteria met during worst episode. We use the term symptoms to refer to the 13 disaggregated DSM-III-R criteria. 
Familial influences on the clinical characteristics of major depression

Twin correlations for any variable are perforce limited by the reliability of that variable. To assess this reliability, we compared the available clinical features of MD for the 54 twins who met criteria both for the year prior to the first and the year prior to the second interview. These individuals were assessed using the identical interview format at a mean interval of 17 months. This analysis jointly assesses both the reliability of experiencing the clinical features of depression over separate episodes and the reliability of reporting them. It therefore differs from the other previous examination of this issue (19), which examines only the latter.

\section{Statistical analysis}

For continuous measures, we calculated the productmoment correlations. We also repeated these after transformation to reduce skewness and found only insignificant changes. For dichotomous or polychotomous variables, we calculated tetrachoric and polychoric correlations, respectively, using the program PRELIS II (20). As we a priori expect only positive correlations, we present one-tailed $P$ values. The differences between the observed correlations in $\mathrm{MZ}$ and $\mathrm{DZ}$ twins were tested by a chi-square difference test with one degree of freedom using the program LISREL (21) in which we allowed the two correlations to differ and then constrained them to equality. In comparing the $\mathrm{MZ}$ and $\mathrm{DZ}$ correlations, we used a one-tailed test and do not report significance if the results ran counter to expectation (correlations higher in $\mathrm{DZ}$ twins). We consider these analyses exploratory and therefore make no attempt at correction for multiple tests.

\section{Results}

Total sample of concordant pairs

We began by examining the 176 twin pairs where both members reported one or more lifetime episodes of MD (Table 1, first column). In these pairs, age at onset $(r=0.34)$ and treatment seeking $(r=0.25)$ were correlated $(P<0.001$ for both). Degree of impairment $(r=0.14)$ and number of symptoms $(r=0.14)$ were also correlated but at a substantially lower level $(P<0.05)$. Duration of longest episode and number of episodes, measured as a continuous variable or dichotomized into those with recurrent $v s$ nonrecurrent illness, were effectively uncorrelated in pairs of affected twins.

Of the 13 symptoms of MD examined, twin pairs concordant for one or more lifetime episodes of MD were significantly correlated for 5 of them: loss of interest or pleasure $(r=0.22)$, weight gain $(r=0.28)$, increased appetite $(r=0.33)$, psychomotor retardation $(r=0.18)$ and suicidal ideation $(r=0.25)$. Sim-
Table 1. Similarity in concordant twin pairs and test-retest reliability for the clinical features and symptoms of major depression

\begin{tabular}{|c|c|c|c|c|c|}
\hline Variable & $\begin{array}{c}\text { All } \\
\text { twin pairs } \\
\text { concordant } \\
\text { for } \mathrm{MD} \\
n=176\end{array}$ & $\begin{array}{c}M Z \\
\text { twin pairs } \\
\text { concordant } \\
\text { for MD } \\
n=103\end{array}$ & $\begin{array}{c}D Z \\
\text { twin pairs } \\
\text { concordant } \\
\text { for } \mathrm{MD} \\
n=73\end{array}$ & $\begin{array}{l}\text { MZ-DZ } \\
\text { difference }\end{array}$ & $\begin{array}{c}\text { Test- } \\
\text { retest } \\
\text { reliablity } \\
n=54\end{array}$ \\
\hline \multicolumn{6}{|l|}{ Clinical features } \\
\hline Age at onset & $+0.34^{\#}$ & $+0.20^{\dagger}$ & $+0.48^{\#}$ & - & $\sim$ \\
\hline Longest duration & +0.03 & -0.12 & +0.03 & - & $+0.38^{f}$ \\
\hline Impairment & $+0.14^{\dagger}$ & $+0.25^{\neq}$ & +0.02 & * & $+0.59^{\#}$ \\
\hline Number of episodes & +0.03 & 0.00 & $+0.22^{\dagger}$ & - & $+0.28^{\dagger}$ \\
\hline Recurrence & +0.08 & $+0.26^{\neq}$ & -0.31 & \# & +0.06 \\
\hline Treatment-seeking & $+0.25^{\#}$ & $+0.18^{\dagger}$ & $+0.34^{\neq}$ & - & -0.03 \\
\hline Number of symptoms & $+0.14^{\dagger}$ & $+0.20^{\dagger}$ & +0.08 & NS & -0.07 \\
\hline \multicolumn{6}{|l|}{ Symptoms } \\
\hline \multicolumn{6}{|l|}{ Loss of interest or } \\
\hline pleasure & $+0.22^{\neq}$ & +0.11 & $+0.38^{\#}$ & - & $\sim$ \\
\hline Weight loss & $+0.12^{*}$ & $+0.26^{f}$ & -0.05 & t & $+0.39^{f}$ \\
\hline Decreased appetite & 0.00 & +0.12 & -0.15 & $t$ & $+0.18^{*}$ \\
\hline Weight gain & $+0.28^{\#}$ & $+0.41^{\#}$ & -0.01 & $\neq$ & $+0.51^{\#}$ \\
\hline Increased appetite & $+0.33^{\#}$ & $+0.36^{\#}$ & $+0.27^{f}$ & NS & +0.02 \\
\hline Insomnia & 0.06 & $+0.25^{\neq}$ & -0.31 & $\#$ & $+0.53^{\#}$ \\
\hline Hypersomnia & 0.06 & $+0.22^{\dagger}$ & -0.30 & $\#$ & $+0.34^{\neq}$ \\
\hline Agitation & -0.04 & -0.03 & -0.06 & NS & $+0.23^{\dagger}$ \\
\hline Retardation & $+0.18^{\neq}$ & $+0.13^{\dagger}$ & $+0.25^{\dagger}$ & - & +0.05 \\
\hline Fatigue & -0.07 & -0.10 & -0.02 & - & -0.22 \\
\hline Worthlessness/guilt & +0.01 & -0.03 & +0.09 & - & $+0.43^{\#}$ \\
\hline Diificulty concentrating & $+0.10^{*}$ & +0.28 & -0.10 & $\neq$ & +0.20 \\
\hline Suicidal ideation & $+0.25^{\#}$ & $+0.32^{\#}$ & $+0.13^{*}$ & * & $+0.70^{\#}$ \\
\hline
\end{tabular}

- Against one-tailed prediction, no $P$ value reported. $P$ values (one-tailed): ${ }^{*} P<0.10$; † $P<0.05 ;{ }^{\neq} P<0.01$; ${ }^{\#} P<0.001, \sim$ Not assessed or not assessable due to too little variance.

ilarity in these twin pairs for weight loss $(r=0.12)$ and difficulty concentrating $(r=0.10)$ were present at the trend level only.

\section{Concordant $\mathrm{MZ}$ and $\mathrm{DZ}$ twin pairs}

The sample contained $103 \mathrm{MZ}$ and $73 \mathrm{DZ}$ twin pairs concordant for the presence of one or more episodes of MD by DSM-III-R criteria. Concordant MZ twin pairs were significantly correlated for 5 of the 7 clinical features of MD: age at onset $(r=0.20)$, impairment $(r=0.25)$, recurrence $(r=0.26)$, treatmentseeking $(r=0.18)$ and total number of symptoms $(r=0.14)$. Although significantly correlated for recurrence, $\mathrm{MZ}$ twins were entirely uncorrelated for the total number of lifetime episodes. DZ twin pairs were significantly correlated for 3 clinical features of MD: age at onset $(r=0.48)$, number of episodes $(r=0.22)$ and treatment-seeking $(r=0.34)$. The correlation in concordant $\mathrm{MZ}$ twin pairs significantly exceeded that found in $\mathrm{DZ}$ pairs only for recurrence and, at a trend level, for degree of impairment.

Concordant $\mathrm{MZ}$ twin pairs were significantly and positively correlated for 7 of the 13 individual symptoms, compared with 3 in the concordant DZ twin 
pairs. Five of the 7 significant correlations in $\mathrm{MZ}$ twin pairs were from vegetative symptoms reflecting depression-related changes in appetite, weight and sleep. In 7 symptoms (weight loss, decreased appetite, weight gain, insomnia, hypersomnia, trouble concentrating and suicidal ideation), of which 5 were vegetative, the correlations in concordant $M Z$ twin pairs significantly exceeded those found in DZ twin pairs.

\section{Reliability of clinical features and symptoms}

Twin correlations for a variable are, per force, limited by the degree of measurement error. In general, twins should not correlate more highly with one another than individuals would correlate with themselves over time. Our sample contained 54 individuals who reported one or more episodes of MD in the last year both at our first and second interviews. The correlations between the reported clinical features of these episodes are seen in the last column of Table 1. Three of the 6 assessed clinical variables (longest duration, number of episodes in the last year and impairment) were significantly correlated over time whereas, somewhat surprisingly, treatmentseeking and total number of symptoms were not. Of the 12 assessed symptoms, twins significantly correlated with themselves over time in 7 of them, of which 4 were vegetative symptoms. The mean $( \pm S D)$ correlation of all 12 symptoms in concordant MZ twins $(r=0.18 \pm 0.16)$ is about two-thirds as large as the mean correlation of individuals with themselves over time $(r=0.28 \pm 0.26)$.

\section{Discussion}

We sought to understand in this study the degree to which familial factors influence the clinical features and symptoms of MD. We know that MD runs in families, but we know little about whether an individual's family background influences the clinical characteristics of MD they display if they become affected.

We found that age at onset of MD was significantly correlated in all twin pairs where both members had one or more lifetime episodes of MD. The magnitude of this correlation $(r=0.34)$ is within the range previously reported in pairs of first degree relatives by Slater (10) $(r=0.35)$, Gershon et al. (12) $(r=0.40)$ and Maier $(11)(r=0.20)$. Winokur (22) has also reported a significant association between age at onset, dichotomized into early and late, in depressive subjects and their affected relatives. Contrary to expectation, age at onset was more similar in concordant $\mathrm{DZ}$ than in concordant $\mathrm{MZ}$ twins. This pattern of findings suggests that the similarity of age at onset of MD in pairs of relatives is prob- ably due to familial-environmental and not genetic factors.

Although the correlation in age at onset of MD in relatives was relatively modest, these results must be interpreted in the context of the reliability of this variable. Bromet et al. (19) found the test-retest reliability of age at first episode to be $r=0.51$ in a community sample. If generalizable, this would suggest that $60 \%$ or more of the reliable variance in age at onset of MD may be due to familial factors.

The degree of impairment during the worst episode of MD was modestly correlated in all concordant twin pairs and, at a trend level, more significantly correlated in $\mathrm{MZ}$ than in $\mathrm{DZ}$ twins. This feature was also one of the most stable across time within the same individual. The correlation in the degree of impairment in concordant $\mathrm{MZ}$ twins was about $40 \%$ as large as that from the same individual measured twice. We know of no previous report that has examined resemblance in relatives for this clinical feature of MD. In this sample (23) and in the one previous study to examine this question (6), degree of impairment was a strong predictor of the risk of MD in co-twins. These results, taken together, tentatively suggest that the degree of impairment may be an index of the genetic liability to MD.

The total numbers of lifetime episodes were not significantly correlated in all or in $\mathrm{MZ}$ twin pairs. However, treated as a dichotomy of recurrencenonrecurrence, concordant $\mathrm{MZ}$ twin pairs were significantly correlated, and their correlation significantly exceeded that found in DZ twins. These results are consistent with those of Maier et al. (11), who found that the risk for recurrent depression was substantially higher in relatives of subjects with recurrent $v s$ nonrecurrent MD. Our findings are less compatible with those from Slater's twin-family study in which he found no substantial resemblance for recurrence rates in affected relatives (10). Our results are consistent with the hypothesis that, given depressive illness, genetic factors may influence whether a recurrent or nonrecurrent variety occurs. However, given the recurrent form, the actual number of episodes experienced may be influenced largely by environmental experiences not shared in twin pairs.

Treatment-seeking was highly significantly correlated in concordant twin pairs. The correlations for help-seeking were actually higher in DZ than in MZ twins. Consistent with previous literature, these results suggest that whether an individual seeks professional help, given a state of depression, is influenced by family background and not genetic factors. Although we are unaware of previous studies that have explicitly addressed this question, 2 studies have reported results that are broadly relevant. In McGuffin's recently published twin study of MD, 
the diagnosis of MD required treatment-seeking (24). Although the estimated heritability of liability to MD $(51 \%)$ was similar to the 2 other comparable twin studies $(15,25)$, the estimated proportion of liability due to familial environment $(31 \%)$ was much higher. When McGuffin completed a blind diagnosis of this same sample when treatment was no longer a diagnostic criteria for $\mathrm{MD}$, the estimated proportion of variance due to the family environment dropped to zero (P. McGuffin, personal communication, 11/91). $\mathrm{He}$ suggested that the probability of help-seeking given MD might be influenced by familial environmental factors. A similar conclusion was reached from the Stockholm adoption study, in which diagnoses were based on sick leave and medical records (26). Here, the investigators found a significant relationship between the risk of mental illness in adopted children and their adoptive parents, but it was not specific to any diagnosis. They suggested that this pattern may result from the non-genetic parent offspring transmission of help-seeking behavior.

The longest duration of an episode of MD was not significantly correlated in concordant twin pairs. Slater also found no significant resemblance for the duration of affective illness in pairs of affected relatives from his twin-family study (10).

Overall, the resemblance of pairs of affected twins for individual depressive symptoms was quite modest. All concordant pairs were positively correlated for only 5 of 13 examined, symptoms with an average correlation across all symptoms of only $r=0.11$. Despite these unimpressive correlations, 2 trends are noteworthy. First, in all twin pairs, but especially in concordant $\mathrm{MZ}$ twin pairs, much of the twin similarity was concentrated in symptoms that reflected the vegetative features of MD. Five of the 6 symptoms reflecting depression-related changes in weight, appetite and sleep were significantly correlated in MZ twin pairs concordant for MD. Furthermore, for all 6 vegetative symptoms, concordant MZ twins resembled one another substantially more than concordant DZ twins, and this difference was significant for 5 of them.

This pattern of results would be expected if both classic MD, with the tendency toward decreased appetite, weight loss and insomnia, and atypical MD $(27-29)$, which frequently presents with reverse vegetative features of increased appetite, weight gain and hypersomnia, were each influenced by genetic factors, but the genetic factors for the 2 conditions were at least partly independent.

We are unaware of any previous results that address the similarity of vegetative features in pairs of relatives affected with MD. Of some relevance, however, is the finding of Leckman et al. (13) that the risk for endogenous MD is substantially higher in relatives of subjects with endogenous vs nonendogenous MD. Three of the 10 symptomatic criteria for endogenous MD in their study involved insomnia, poor appetite and weight loss (30).

Suicidal ideation was significantly correlated in all concordant twin pairs and was substantially and marginally significantly higher in $\mathrm{MZ} v s \mathrm{DZ}$ twin pairs. These results are consistent with previous evidence from family and adoption studies that suicide might itself be influenced by familial factors that are, at least in part, probably genetic (31).

These modest correlations in individual symptoms in pairs of concordant twins must be interpreted in the context of the reliability of such assessments. We are unaware of specific studies of such symptoms (although investigators have examined the stability of psychotic symptoms in MD (32)). In our sample, the mean tetrachoric correlation in symptoms for individuals who reported episodes of depression in two different years was only $r=0.28$. It is therefore possible that familial factors account for a substantial proportion of the variance in the symptoms of MD that, in any individual, can be expected to be stable over time.

Birnbaum (33) suggested that clinical characteristics of mental disorders could be divided into pathogenic and pathoplastic. He defined pathogenic clinical features as "... those which refer to the actual cause of the illness, which endow it with a specific characters, its quality of being thus and no other". Pathoplastic features are, by contrast, "those which may be said to shape the disorder in that they give content, colouring, and contour to individual illnesses whose basic form and character have already been biologically established".

One way in which to view the results of this study is as an attempt, in the context of Birnbaum's theory, to determine which clinical features of the broad syndrome of MD are pathogenic (influenced by familial and especially genetic factors) versus pathoplastic (determined by idiosyncratic non-familial factors). Our results leave considerable room for the operation of both sets of factors. On the one hand, the clinical features of MD were not, in our sample, randomly distributed in concordant twin pairs, as would be expected if they were all pathoplastic arising from idiosyncratic personal factors. On the other hand, twin resemblance for these clinical features was modest at best, even in concordant $\mathrm{MZ}$ twins, suggesting that individual or pathoplastic features are also quite important in the syndrome of MD.

These results should be interpreted in the context of 5 potentially significant methodologic limitations. First, because of its low prevalence (34) and low reliability in general population samples (35), mania was not assessed in our interviews. Therefore, we cannot distinguish individuals whose MD occurred 


\section{Kendler et al.}

in the setting of unipolar illness from those with bipolar illness. From previous prevalence figures, it can be estimated that bipolar cases should constitute no more than $5-10 \%$ of our sample of twins with MD. It is possible that some of the observed familial aggregation of symptoms of MD result from the tendency for bipolar illness to itself run in families (1).

Second, the lifetime prevalence rate for MD is higher in our sample than in several recent epidemiologic studies using similar diagnostic criteria $(36,37)$. However, these studies used lay-interviewers and a highly structured psychiatric interview (38), a procedure that may underestimate the population rates of MD (39). Before our probe questions for lifetime MD, we included a statement emphasizing the need for the respondents to take time to think back over their entire life before answering. This may have increased our ascertainment rate for prior episodes of MD because our ratio of lifetime to oneyear prevalence in our first interview (3.3:1) is much higher than that obtained in women in the Epidemiologic Catchment Area study (1.75:1) (36). In addition, the younger age of our twins compared with that of most previous population samples may, because of a cohort effect (40), also be partly responsible for the high observed prevalence rates. Finally, several recent population-based studies, including at least one with very similar methods (41), report lifetime prevalence rates for MD in women that are as high or higher than those reported here $(42,43)$.

Could being a twin be associated with an increased rate of MD? Previous studies have not found different rates of mental disorder in general or depression in particular in twins vs singletons (44-46). MZ twins are, compared with DZ twins, generally more aware of and more affected by their twinness. If twinness were associated with MD, one might predict that MZ twins should have higher rates of MD than DZ twins, a pattern that is not found in the present samples (15).

Third, although we began with a large sample of twins, the number of pairs concordant for a lifetime history of MD is relatively modest, especially when divided by zygosity. Because of this small sample and the methodologic issues outlined below, we did not attempt formal model fitting, instead limiting ourselves to qualitative judgments about the relative magnitude of correlations in $\mathrm{MZ} v s \mathrm{DZ}$ twins.

Fourth, many tests were performed in these analyses without strong guiding a priori predictions. Although the number of significant findings clearly exceeded chance expectation, some of the significant findings are probably the result of chance fluctuations. Only replication can definitively resolve which of our findings are likely to be true.

Finally, as we have explored in detail elsewhere $(47,48)$, the causes of familial resemblance for dis- ease traits can be quite complex and may arise from at least 3 different mechanisms. First, a trait can be correlated in relatives because it is an index of disease liability that is itself familial. For example, if severity of impairment reflects the overall liability to MD, then pairs of affected relatives will be correlated for their degree of impairment because they are correlated for their overall disease liability. However, in this situation, the observed correlation for such a trait in relatives will be attenuated due to the truncation in the range of disease liability that arises from examining only affected individuals. Furthermore, if the liability is genetically influenced, this truncation effect will differ in $\mathrm{MZ}$ and $\mathrm{DZ}$ twins. Although a full model-fitting treatment of this problem is available for uncensored data (47), current software can not handle the differences in years at risk in the present sample.

Second, disease traits may be correlated in affected relatives because they are influenced by familial factors unrelated to disease liability. For example, certain familial personality characteristics may be uncorrelated with the liability to depression but, given that an individual is depressed, can influence the clinical features of that disorder.

Third, etiologic heterogeneity could also result in correlated disease traits in pairs of affected relatives. If typical and atypical MD were different disorders but both familial, pairs of relatives would tend to have the same form of illness. Therefore, clinical features that differed in the two forms of depression (such as patterns of eating and sleeping) would be correlated in affected relatives.

Although the problem is a formidable one, we hope in the future to develop a more complete modelbased approach to both detecting the presence and determining the possible cause of familial resemblance for symptoms of mental disorders such as MD. Because of the modest correlations observed, the present sample size of pairs of twins concordant for MD is, however, unlikely to permit a definitive resolution of these issues.

\section{Acknowledgements}

This work was supported by grant MH-40828 from the United States National Institutes of Mental Health. The Virginia Twin Registry, established and maintained by W. Nance MD, PhD and L. Corey $\mathrm{PhD}$, is supported by the United States National Institutes of Health grants HD-26746 and NS-25630. Leroy Thacker MS assisted in the data analysis.

\section{References}

1. Tsuang MT, Faraone SV. The genetics of mood disorders. Baltimore: Johns Hopkins University Press, 1990.

2. Weissman MM, Merikangas KR, Wickramaratne P et al. Understanding the clinical heterogeneity of major depression using family data. Arch Gen Psychiatry 1986: 43: $430-434$. 
3. Stancer HC, Persad E, Wagener DK, Jorna T. Evidence for homogeneity of major depression and bipolar affective disorder. J Psychiatr Res 1987: 21: 37-53.

4. McGuffin P, Katz R, Bebbington P. Hazard, heredity and depression: a family study. J Psychiatr Res 1987: 21: 365-375.

5. Gershon ES, Weissman MM, Guroff JJ, Prusoff BA, LECKMAN JF. Validation of criteria for major depression through controlled family study. J Affective Disord 1986: 11: 125-131.

6. Bland RC, Newman SC, Orn H. Recurrent and nonrecurrent depression: a family study. Arch Gen Psychiatry 1986: 43: $1085-1089$.

7. Leckman JF, Caruso Ka, Prusoff BA, Weissman MM, Merikangas KR, Pauls DL. Appetite disturbance and excessive guilt in major depression: use of family study data to define depressive subtypes. Arch Gen Psychiatry 1984: 41: 839-844.

8. KLEIN DN. Symptom criteria and family history in major depression. Am J Psychiatry 1990: 147: 850-854.

9. Grove WM, andreasen NC, Winokur G, Clayton PJ, Endicott J, Coryell WH. Primary and secondary affective disorders: unipolar patients compared on familial aggregation. Compr Psychiatry 1987: 28: 113-126.

10. Slater E. Psychotic and neurotic illnesses in twins. London: Her Majesty's Stationery Office, 1953.

11. Maier W, Lichtermann D, Minges J, Heun R, HallMAYER J, KLINGLER T. Unipolar depression in the aged: determinants of familial aggregation. J Affective Disord 1991: 23: $53-61$.

12. Gershon ES, Hamovit J, Guroff JJ et al. A family study of schizoaffective, bipolar I, bipolar II, unipolar, and normal control probands. Arch Gen Psychiatry 1982: 39: 1157-1167.

13. Leckman JF, Weissman MM, Prusoff BA et al. Subtypes of depression: family study perspective. Arch Gen Psychiatry 1984: 41: 833-838.

14. American Psychiatric Association. Diagnostic and statistical manual of mental disorders. 3rd edn., revised. Washington, DC: APA, 1987.

15. Kendler KS, Neale MC, Kessler RC, Heath aC, EAVES LJ. A population based twin study of major depression in women: the impact of varying definitions of illness. Arch Gen Psychiatry 1992: 49: 257-266.

16. Eaves LJ, Eysenck HJ, Martin NG et al. Genes, culture and personality: an empirical approach. London: Oxford University Press, 1989.

17. Spence JE, Corey La, Nance We, Marazita ML, KendLer KS, SCHIEKeN RM. Molecular analysis of twin zygosity using VNTR DNA probes. Am J Hum Genet 1988: 43 (3): A159.

18. Spitzer RL, Williams JB, Gibbon M. Structured Clinical Interview for DSM-III-R. New York: Biometrics Research Department, New York State Psychiatric Institute, 1987.

19. Bromet EJ, Dunn Lo, Connell MM, Dew MA, SchulBERG HC. Long-term reliability of diagnosing lifetime major depression in a community sample. Arch Gen Psychiatry 1986: 43: $435-440$.

20. JoRESKOG KG, SorboM D. PRELIS: a preprocessor for LISREL. Mooresville, IN: Scientific Software, 1988.

21. JorESKoG KG, Sorbom D. LISREL 7: a guide to the program and applications. 2nd edn. Chicago, IL: SPSS, 1989.

22. Winokur G, Cadoret RJ, Baker M, Dorzab J. Depression spectrum disease versus pure depressive disease: some further data. Br J Psychiatry 1975: 127: 75-77.

23. Kendler KS, Neale MC, Kessler RC, Heath aC, EAves LJ. The characteristics of familial depression. Submitted.

24. McGuffin P, Katz R, Rutherford J. Nature, nurture and depression: a twin study. Psychol Med 1991: 21: 329335.

25. Torgersen S. Genetic factors in moderately severe and mild affective disorders. Arch Gen Psychiatry 1986: 43: 222226.

26. von Knorring AL, Cloninger CR, Bohman M, SigVARDSSON S. An adoption study of depressive disorders and substance abuse. Arch Gen Psychiatry 1983: 40: 943-950.

27. Paykel ES, Rowan PR, Rao B, Bhat A. Atypical depression: nosology and response to antidepressants: In: Clayton P Barrett J, ed. Treatment of depression: old controversies and new approaches. New York: Raven Press, 1983: 237-251.

28. Davidson J, Miller R, Turnbull C, Sullivan J. Atypical depression. Arch Gen Psychiatry 1982: 39: 527-534.

29. Quitkin FM, Stewart JW, MCGrath PJ et al. Phenelzine versus imipramine in the treatment of probable atypical depression: defining syndrome boundaries of selective MAOI responders. Am J Psychiatry 1988: 145: 306-311.

30. Spitzer RL, Endicott J, Robins E. Research Diagnostic Criteria for a selected group of functional disorders. 2nd edn. New York: New York Psychiatric Institute, 1975.

31. Roy A. Genetics and suicidal behavior. In: Pfeffer C, ed. Suicide among youth: perspectives on risk and prevention. Washington, DC: American Psychiatric Association Press, 1989: 165-183.

32. Helms PM, Smith RE. Recurrent psychotic depression: evidence of diagnostic stability. J Affective Disord 1983: 5: $51-54$.

33. BiRnBaum K. The making of a psychosis: the principles of structural analysis in psychiatry. In: HIRSCH SR, SHEPHERD $M$, ed. Themes and variations in European psychiatry: an anthology. Charlottesville: University Press of Virginia, 1974: 199-238.

34. Boyd JH, Weissman MM. Epidemiology of affective disorders: a reexamination and future directions. Arch Gen Psychiatry 1981: 38: 1039-1046.

35. Andreasen NC, Grove WM, Shapiro RW, Keller MB, Hirschfeld RMA, McDonald-Scott P. Reliability of life-time diagnoses. Arch Gen Psychiatry 1981: 38: 400-405.

36. Weissman MM, Bruce ML, Leaf PJ, Florio LP, Holzer C. Affective disorders. In: RobINS LN, REgIer DA, ed. Psychiatric disorders in America: the Epidemiologic Catchment Area Study. New York: Free Press, 1991: 53-80.

37. Wells JS, Bushnell FA, Hornblow AR, Joyce PR, OAKLEY-BROWNE MA. Christchurch psychiatric epidemiology study. I. Methodology and lifetime prevalence for specific psychiatric disorders. Aust NZ J Psychiatry 1989: 23: 315326.

38. Robins LN, Helzer JE. Diagnostic Interview Schedule (DIS): version III-A. St. Louis, MO: Washington University School of Medicine, 1985.

39. PARKer G. Are the lifetime prevalence estimates in the ECA study accurate? Psychol Med 1987: 17: 275-282.

40. Klerman GL, Lavori PW, Rice J, Reich T, EndicotT J, ANDREASEN NC. Birth-cohort trends in rates of major depressive disorder among relatives of patients with affective disorder. Arch Gen Psychiatry 1985: 42: 689-693.

41. Weissman MM, Myers JK. Affective disorders in a US urban community: the use of Research Diagnostic Criteria in an epidemiological survey. Arch Gen Psychiatry 1978: 35: 1304-1311.

42. Rorsman B, Grasbeck A, Hagnell $O$ et al. A prospective study of first-incidence depression: the Lundby study, 1957-1972. Br J Psychiatry 1990: 156: 336-342.

43. Bebbington P, Katz R, McGuffin P, Tennant C, HurRY J. The risk of minor depression before age 65 : results from a community survey. Psychol Med 1989: 19: 393-400. 


\section{Kendler et al.}

44. Kringlen E. Heredity and environment in the functional psychoses: an epidemiological-clinical twin study. Oslo: Universitetsforlaget, 1967.

45. Paluszny M, Selzer ML, Vinokur A, Lewandowski L. Twin relationships and depression. Am J Psychiatry 1977: 134: 988-990.

46. Chitkara B, MacDonald A, Reveley AM. Twin birth and adult psychiatric disorder: an examination of the case records of the Maudsley hospital. Br J Psychiatry 1988: 152: 391-398.

47. Neale MC, Eaves LJ, Hewitt JK, Meyer JM, KenDLER KS. Analyzing the relationship between age of onset and risk to relatives. Am J Hum Genet 1989: 45: 226-239.

48. Kendler KS, Tsuang MT, Hays P. Age at onset in schizophrenia: a familial perspective. Arch Gen Psychiatry 1987: 44: 881-890. 